\title{
Modified iterative versus Laplacian Landau gauge in compact U(1) theory
}

\author{
Stephan Dürr \\ DESY Zeuthen, 15738 Zeuthen, Germany, \\ INT at University of Washington, Seattle, Washington 98195-1550, \\ and PSI Villigen, 5232 Villigen PSI, Switzerland \\ Philippe de Forcrand \\ ETH Zürich-Hönggerberg, 8093 Zürich, Switzerland \\ and CERN, Theory Division, 1211 Geneva 23, Switzerland
}

(Received 4 July 2002; published 25 November 2002)

\begin{abstract}
Compact U(1) theory in four dimensions is used to compare the modified iterative and Laplacian fixing to the lattice Landau gauge in a controlled setting, since in the Coulomb phase the lattice theory must reproduce the perturbative prediction. It turns out that on either side of the phase transition clear differences show up and in the Coulomb phase the ability to remove double Dirac sheets proves vital on a small lattice.
\end{abstract}

DOI: 10.1103/PhysRevD.66.094504

\section{INTRODUCTION}

One of the main practical advantages one exploits in Monte Carlo studies of lattice QCD is the fact that phenomenologically relevant low-energy observables (i.e., masses and matrix elements of operators between physical states) may be studied without a need to choose a specific gauge; i.e., they may be determined from gauge-invariant correlators, and hence the functional integral may perform a random sampling over all gauge orbits.

Nonetheless, there are good reasons to address the question of how one may transform a given ensemble of gauge configurations to a specific gauge; mainly because on such a gauge-fixed ensemble gauge-dependent objects such as quark or gluon propagators may be determined too, and the results may be compared with what one gets in perturbation theory or in other nonperturbative frameworks, such as the Dyson-Schwinger approach.

An attempt to fix a given configuration to a specific gauge raises several issues. The first question to ask is what a lattice analogue of a continuum gauge such as the Lorentz condition

$$
\partial_{\mu} A_{\mu}=0,
$$

might be, and the typical answer is to construct a functional on the space of lattice configurations such that its extremum simplifies in the continuum limit to Eq. (1), but the choice is, of course, not unique. The simplest option is the standard lattice Landau gauge functional

$$
F[\{U\}]=\frac{1}{8 V} \sum_{x, \mu}\left(1+\frac{1}{N_{c}} \operatorname{Re} \operatorname{Tr}\left[U_{\mu}^{\Omega}(x)\right]\right)
$$

where $U_{\mu}^{\Omega}(x)=\Omega(x)^{\dagger} U_{\mu}(x) \Omega(x+\hat{\mu})$ is the gauge transformed link variable and $V=\Sigma_{x} 1$, but it may be worthwhile to maximize an "improved" functional instead (see, e.g., [1]). The second (more technical) question is which procedure will be used to search for the maximum ${ }^{1}$ of a given

\footnotetext{
${ }^{1}$ If there is a unique extremum at all, i.e., if there are no genuine Gribov copies [2].
}

PACS number(s): 11.15.Ha

functional like Eq. (2), since the latter represents a very highdimensional extremization problem, and there is, unfortunately, no known algorithmic solution.

In view of this problem, Laplacian Landau gauge fixing has been proposed [3] - a method that does not directly attempt to extremize Eq. (2) but rewrites the problem such that, after a set of local constraints is traded for a global one, the latter task may be solved exactly. The advantage is that the procedure is unique, i.e., the final configuration does not depend on the initial gauge. It has led to beautiful results in quenched SU(2), SU(3), and SU(4) studies [4], but since there is no firm analytic prediction to compare with, the situation is not conclusive. As a result, a good fraction of the research that tries to link the QCD confinementdeconfinement phenomenon to the behavior of specific oneor two-dimensional defect structures (e.g., monopoles or vortices) deals with algorithmic issues and there is, to date, no agreement on the physics lessons learned.

In this situation, we decided to address the question in a simpler setting, namely, the pure compact U(1) gauge theory in four dimensions with the standard Wilson action

$$
\begin{aligned}
S[\{U\}]= & \beta \sum_{x, \mu<\nu}\left\{1-\operatorname{Re}\left[U_{\mu}(x) U_{\nu}(x+\hat{\mu})\right.\right. \\
& \left.\left.\times U_{\mu}(x+\hat{\nu})^{\dagger} U_{\nu}(x)^{\dagger}\right]\right\} \\
= & \beta \sum_{\square}\left[1-\cos \left(\theta_{\square}\right)\right]
\end{aligned}
$$

where $\beta=1 / g^{2}, U_{\mu}(x)=\exp \left(\mathrm{i} \theta_{x, \mu}\right) \in U(1)$, and $\theta_{\square}=\theta_{x, \mu \nu}$ $=\theta_{x, \mu}+\theta_{x+\hat{\mu}, \nu}-\theta_{x+\hat{\nu}, \mu}-\theta_{x, \nu}$ is the plaquette angle. This theory is known to have two phases [5]: For $\beta>\beta_{\mathrm{c}}$ it is in the ordered ("Coulomb") phase; for $\beta<\beta_{\mathrm{c}}$ it is in the disordered ("confined") phase. Contrary to what early and some more recent studies indicate [6], the transition seems to be first order [7] with $\beta_{\mathrm{c}} \simeq 1.01$.

What is important in our context is that the $\mathrm{U}(1)$ theory, if quantized on the torus $T^{4}$, shows the Gribov ambiguity mentioned above [8]. Several numerical studies have tried to pin down the effect gauge fixing artifacts have on the mass of the "photon" in either phase. First, Coddington et al. studied the transverse zero-momentum correlator 


$$
C(t)=\sum_{\mathbf{x}, \mathbf{y}} \sum_{i=1}^{3} A_{i}(\mathbf{x}, 0) A_{i}(\mathbf{y}, t)
$$

where they defined the photon field (and we follow them in this choice) through

$$
A_{\mu}(x)=\operatorname{Im} U_{\mu}(x)=\sin \left(\theta_{x, \mu}\right)
$$

and they found that—at zero momentum - the "photon" behaves as a massless particle for $\beta>\beta_{\mathrm{c}}$ but as a massive one at $\beta<\beta_{\mathrm{c}}$ [9]. Thereby they confirmed an earlier result, obtained in a gauge-invariant approach [10]. Nakamura and Plewnia extended this work to arbitrary spatial momentum, i.e., they measured the correlator

$$
C_{\mu \nu}(\mathbf{p}, t)=\sum_{\mathbf{x}, \mathbf{y}} A_{\mu}(\mathbf{x}, 0) A_{\nu}(\mathbf{y}, t) e^{-\mathbf{i} \cdot(\mathbf{x}-\mathbf{y})}
$$

for a few low-lying $\mathbf{p}$ and observed that the transverse part at $\beta=1.1$ deviates, for large time separations, quite drastically from the perturbative prediction, if Eq. (2) is maximized through local iterative steps only [11]. They also noticed that the result improves, if the local iterative procedure is extended to opt for the best one out of several attempts, and this observation triggered a long stream of subsequent activities (see below).

The goal of the present study is to give a fair comparison between Laplacian and iterative gauge fixing in the pure $\mathrm{U}(1)$ theory, where both phases may be considered, good statistics is easy to obtain, and correlators involving all momenta may be determined through fast Fourier transform. After a quick review of the gauge-fixing procedures, the comparison is given for the Coulomb and confined phases separately, and we end with a short discussion of the relevance of our results to the non-Abelian case.

\section{ITERATIVE VERSUS LAPLACIAN GAUGE FIXING}

We start with a brief outline of the two gauge fixing algorithms which we are going to compare with respect to the photon correlators implied.

\section{A. Standard and modified iterative Landau gauge fixing}

Modified iterative Landau gauge fixing is an improvement on standard iterative Landau gauge fixing. The latter is tantamount to iteratively passing through the lattice and trying to locally maximize the gauge functional (2), which in the case of the compact $\mathrm{U}(1)$ theory reads

$$
\begin{aligned}
F[\{\theta\}] & =\frac{1}{V} \sum_{x} F_{x}(\theta), \\
F_{x}(\theta) & =\frac{1}{16} \sum_{\mu=1}^{4}\left[2+\cos \left(\theta_{x, \mu}\right)+\cos \left(\theta_{x-\hat{\mu}, \mu}\right)\right] .
\end{aligned}
$$

In this symmetrized version it is obvious that for a given site $x$ the local gauge transformation

$$
\Omega(x): \theta_{x, \mu} \rightarrow \theta_{x, \mu}+c_{x}, \theta_{x-\hat{\mu}, \mu} \rightarrow \theta_{x-\hat{\mu}, \mu}-c_{x}
$$

that maximizes $F_{x}(\theta)$ may be found analytically. Moreover, doubling the respective $c$ leaves the local contribution $F_{x}(\theta)$ unchanged, thus offering the possibility of an overrelaxation step: in practice one sweeps through the lattice in a mixed overrelaxation and maximization strategy [12].

In general, this standard procedure gets stuck in a local extremum. If the common belief that the "true" gauge copy corresponds to the global maximum [13] is correct, an extra effort is needed to improve on this situation. Specifically, for the case of the compact U(1) theory two types of objects are known which prevent the local iterative procedure from reaching the true extremum. Bogolubsky et al. have shown [14] that in the quest for the global maximum one needs to suppress both the "double Dirac sheets" (DDS's) and the "zero-momentum modes" (ZMM's) of the gauge field, two concepts which we shall explain in detail.

1. DDSs: Each plaquette angle $\theta_{x, \mu \nu}$ is decomposed into the gauge-invariant electromagnetic flux $\left.\bar{\theta}_{x, \mu \nu} \in\right]-\pi, \pi[$ and the discrete gauge-dependent part $2 \pi n_{x, \mu \nu}, n_{x, \mu \nu}=0$, $\pm 1, \pm 2$ [15]. A value $n_{x, \mu \nu}= \pm 1$ represents a Dirac string passing through the plaquette and a plaquette with $n_{x, \mu \nu} \neq 0$ is called a "Dirac plaquette." A gauge-invariant monopole inside an elementary cube is detected via its nonzero flux $2 \pi \Sigma_{6} n_{\mu \nu}$. Since monopole worldlines form closed loops on the dual lattice, the Dirac strings normally span cylindrical $2 \mathrm{~d}$ sheets dual to the Dirac plaquettes. However, on a lattice with periodic boundary conditions, it is also possible to form a boundary-free Dirac sheet spanning a whole two-torus. It represents the exceptional case of two monopole loops merging on top of each other. A pair of Dirac sheets with opposite flux orientation carries zero action, and can be removed by a periodic gauge-transformation [16]. It is called a double Dirac sheet. In the confining phase, monopole worldlines percolate [17], and so do Dirac strings and sheets. In the Coulomb phase, monopole loops are small, Dirac plaquettes are less abundant, and DDSs are rare. When they appear, they tend to lie parallel to the main axes, presumably for entropic reasons, since this orientation requires fewer Dirac plaquettes. In any case, a DDS in the $\sigma \rho$ plane may be detected by counting the total number of $\mu \nu$ Dirac plaquettes $\left(\varepsilon_{\mu \nu \sigma \rho} \neq 0\right)$, which must satisfy

$$
N_{\mathrm{DP}}^{(\mu \nu)} \geqslant 2 L_{\sigma} L_{\rho} .
$$

2. ZMMs: With periodic boundary conditions the zeromomentum modes of the gauge field 


$$
\bar{\theta}_{\mu}=\frac{1}{V} \sum_{x} \theta_{x, \mu}
$$

do not contribute to the action (3) - the angle of the Polyakov loop is arbitrary, indicating a global U(1) symmetry of the action. For gauge configurations representing small fluctuations around constant modes it is easy to see that the global extremum of the functional (7) requires $\bar{\theta}_{\mu}=0$. The latter condition may be satisfied through a nonperiodic transformation

$$
\left.\theta_{x, \mu} \rightarrow \theta_{x, \mu}^{c} \equiv \theta_{x, \mu}-c_{\mu} \bmod 2 \pi, c_{\mu} \in\right]-\pi, \pi[,
$$

with the specific choice $c_{\mu}=\bar{\theta}_{\mu}$, but in general this is not a gauge transformation (the Polyakov loop is changed). If one insists that Eq. (11) shall be a gauge transformation, the $c_{\mu}$ need to be chosen from the (finite) set

$$
c_{\mu} \in\left\{0, \pm \frac{2 \pi}{L_{\mu}}, \pm \frac{4 \pi}{L_{\mu}}, \ldots\right\} .
$$

Had we chosen the other natural definition of the gauge field, $A_{\mu}(x)=-i \log \left[U_{\mu}(x)\right]=\theta_{x, \mu}$, rather than (5), the transformation (11) with $c_{\mu}=\bar{\theta}_{\mu}$ would amount to a shift in $A_{\mu}(x)$ such that its constant Fourier mode is zero. In other words, abandoning the restriction (12) amounts to eliminating a constant background field. While this is frequently done (see, e.g., [14]), we feel that sticking with the combination (11), (12), where the background is suppressed but not eliminated, is somewhat closer in spirit to the original extremization taskchoosing, out of Eq. (12), a nontrivial $c_{\mu}$ amounts to switching to another point on the gauge orbit which, in view of the global extremum, looks more promising. In practice, one does not expect any sizable difference between the two options, since on a large enough lattice the discreteness of $c_{\mu}$ turns into a rather marginal restriction.

The "modified iterative Landau gauge" (modified ILG) differs from the "standard" ILG discussed above in that it tries to eliminate both the DDSs and the ZMMs as far as possible. To implement it, we proceed in bunches of 100 standard sweeps through the lattice followed by a ZMM operation (11),(12) and a check on the DDS condition (9) which we sharpen to

$$
N_{\mathrm{DP}}^{(\mu \nu)} \geqslant 1.5 L_{\sigma} L_{\rho}
$$

to account for additional monopole-antimonopole pairs that may punch holes into the sheet. In case Eq. (13) is satisfied, the whole gauge-fixing procedure is started over from another random point on the gauge orbit. This is repeated until a configuration without DDSs is obtained. Further bunches of 100 extremization or overrelaxation sweeps are applied until the last round has increased the functional (7) by less than $10^{-8}$. To prevent the program from getting trapped in an infinite loop, a cutoff on the outer loop is introduced which is chosen $O(30)$ in the Coulomb phase and $O(10)$ in the confined phase (these figures are tuned to our $\beta$ values and lattice size, i.e., they represent an ad hoc element in the procedure). In such a case the result of the random gauge copy which has led to the highest functional value (7) is postiterated with local maximization or overrelaxation steps to make sure that at least a local extremum is reached.

For reasons that will become clear later on, we decided to include the ZMM suppression (11), (12) in our "standard" ILG ensemble, too.

\section{B. Laplacian Landau gauge fixing}

The Laplacian Landau gauge fixing method [3] takes its origin from the observation that maximizing Eq. (7) [we discuss specifically the $\mathrm{U}(1)$ case] is equivalent to minimizing

$$
G[\{\theta\}]=\operatorname{Re}\left(\sum_{x, \mu} 2-e^{i \theta_{x, \mu}-e^{-i \theta_{x-\hat{\mu}, \mu}}}\right),
$$

and the latter means, more explicitly, to search for a gauge transformation (8) such that

$$
\begin{aligned}
G[\{\theta\}]= & \operatorname{Re}\left(\sum_{x, \mu} e^{i c_{x}} 2 e^{-i c_{x}}-e^{i c_{x}} e^{i \theta_{x, \mu}} e^{-i c_{x}+\hat{\mu}}\right. \\
& \left.-e^{i c_{x}} e^{-i \theta_{x}-\hat{\mu}, \mu} e^{-i c_{x}-\hat{\mu}}\right)
\end{aligned}
$$

is minimal. Setting $\phi_{x}=\rho_{x} e^{-i c_{x}}$, the expression in brackets suggestively looks like the quadratic form

$$
\sum_{x, y} \phi_{x}^{\dagger}(-\triangle)_{x y} \phi_{y}
$$

and the problem (15) would, in fact, be equivalent to searching for the lowest eigenmode of the sign flipped covariant Laplacian, were it not for the set of local constraints $\left|\phi_{x}\right|$ $=1\left(\right.$ or $\left.\rho_{x}=1\right)$ for all $x$. The Laplacian Landau gauge [3] ignores the latter and constructs the transformation (8) from the eigenmode that corresponds to the lowest eigenvalue of Eq. (16), i.e., it sets $c_{x}=-\operatorname{Im} \log \left(\phi_{x}\right)$ and keeps only the global constraint $\Sigma_{x}\left|\phi_{x}\right|^{2}=1$ (because the mode may be normalized)

The Laplacian Landau gauge (LLG) is unique, i.e., the final result does not depend on the initial gauge [3], and it is unambiguous in all cases where (a) the lowest eigenmode of the covariant Laplacian is nondegenerate and (b) the result satisfies $\phi_{x} \neq 0$ for all sites $x$. For a continuous (i.e., interpolating) gauge field $\widetilde{A}_{\mu}(\tilde{x})$, the local ambiguities $\widetilde{\phi}(\tilde{x})=0$ are associated with Dirac sheets. For details with respect to (a) and (b) we refer to $[3,4]$ and for a discussion of the relationship of the LLG to the Landau gauge in perturbation theory we refer to [18]. To compute the lowest eigenmode of the covariant Laplacian we use the Arnoldi package ARPACK [19].

\section{COMPARISON IN THE COULOMB PHASE}

We generate 1500 configurations on a $16^{4}$ lattice at $\beta$ $=1.05$, fix them with the Laplacian and the modified iterative prescription, and compare the results for the photon propagator.

A gauge-invariant way to establish, for $\beta>\beta_{\mathrm{c}}$, that the 
photon is massless is to measure the (exponential) decay of the correlator between two planes of spatial Polyakov loops with a few nonzero momenta in the spatial directions. At large enough Euclidean time separation $t$ one determines the effective mass $m_{\text {eff }}$. After introducing the lattice momentum

$$
\hat{p}_{\mu}=\frac{2}{a} \sin \left(\frac{p_{\mu} a}{2}\right)=\frac{2}{a} \sin \left(\frac{\pi n_{\mu}}{L_{\mu}}\right),
$$

plotting $m_{\text {eff }}$ as a function of $\hat{\mathbf{p}}^{2}$, and fitting with

$$
m_{\mathrm{eff}}=\sqrt{m^{2}+\hat{\mathbf{p}}^{2}},
$$

the data ought to be consistent with $m^{2}=0$. Since the available (ordinary) momenta are $\mathbf{p}_{i}=2 \pi \mathbf{n}_{i} / L_{i}$ and good agreement with the dispersion relation (18) typically requires $\mathbf{p}^{2}$ $\ll 1$, the spatial extension of the lattice needs to be large. On our $16^{4}$ lattice, results are acceptable for the two lowest momenta $|\mathbf{p}|=1 \times 2 \pi / 16$ and $|\mathbf{p}|=\sqrt{2} \times 2 \pi / 16$, as displayed in Fig. 1 (top). We fit the correlator with a single and a double cosh function and check whether the two effective masses are consistent with Eq. (18) where the mass has been set to zero, which indeed they are.

Following Nakamura and Plewnia [11], we repeat this in a gauge-dependent way, using our two gauge-fixed ensembles. With that aim we determine the decay properties of the transverse photon-photon correlator (6), again for the same spatial momenta and with indices $\mu=\nu$ which point orthogonal to both $\mathbf{p}$ and the propagation direction (associated with $t$ ). The result is displayed in Fig. 1 (bottom). The important point is that the link-link correlator yields the same information about the photon dispersion relation as the Polyakov loop correlator, but with much higher accuracy. This is easy to understand, since the main effect of the gauge transformations is to "unwind" the Polyakov loop, i.e., to remove multiples of $2 \pi$ which contribute only to the noise.

The main technical point of the present paper is that one may get more detailed information about the performance of either fixing procedure by considering the complete correlator

$$
C_{\mu \nu}(p)=\sum_{x, y} A_{\mu}(x) A_{\nu}(y) e^{-i p(x-y)}=\hat{A}_{\mu}(p) \hat{A}_{\nu}(-p)
$$

for arbitrary four-momentum $p$. Since in the continuum the photon propagator is transverse, it makes sense to split it (on the lattice) into a transverse and a longitudinal piece, viz.,

$$
C_{\mu \nu}(\hat{p})=\left(g_{\mu \nu}-\frac{\hat{p}_{\mu} \hat{p}_{\nu}}{\hat{p}^{2}}\right) D_{\perp}+\frac{\hat{p}_{\mu} \hat{p}_{\nu}}{\hat{p}^{2}} D_{\|}
$$

with

$$
\begin{aligned}
D_{\perp}\left(\hat{p}^{2}\right) & =\frac{1}{3}\left(\operatorname{tr}\left(C_{\sigma \rho}\right)-\frac{\hat{p}_{\sigma} C_{\sigma \rho} \hat{p}_{\rho}}{\hat{p}^{2}}\right), \\
D_{\|}\left(\hat{p}^{2}\right) & =\frac{\hat{p}_{\sigma} C_{\sigma \rho} \hat{p}_{\rho}}{\hat{p}^{2}} .
\end{aligned}
$$
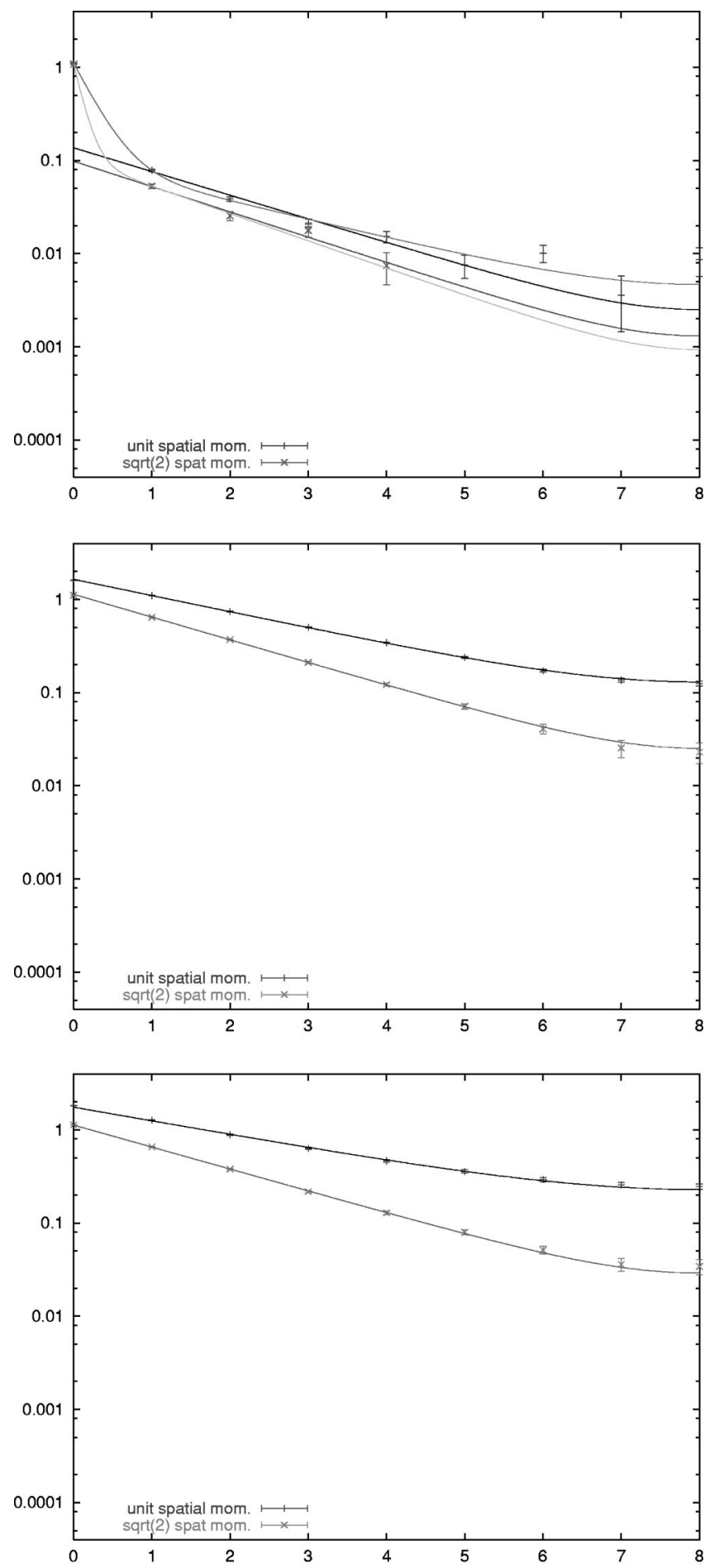

FIG. 1. Top: Correlator between planes of Polyakov loops with once $(+)$ or $\sqrt{2}$ times $(\times)$ the minimum spatial momentum at $\beta$ $=1.05$. From these one might determine two points of the photon dispersion relation (18), but this gauge-invariant estimator is rather noisy. The same information may be obtained, after gauge fixing, with considerably reduced error bars from the photon-photon correlator with the respective momenta in the modified iterative (center) or Laplacian Landau gauge (bottom). 

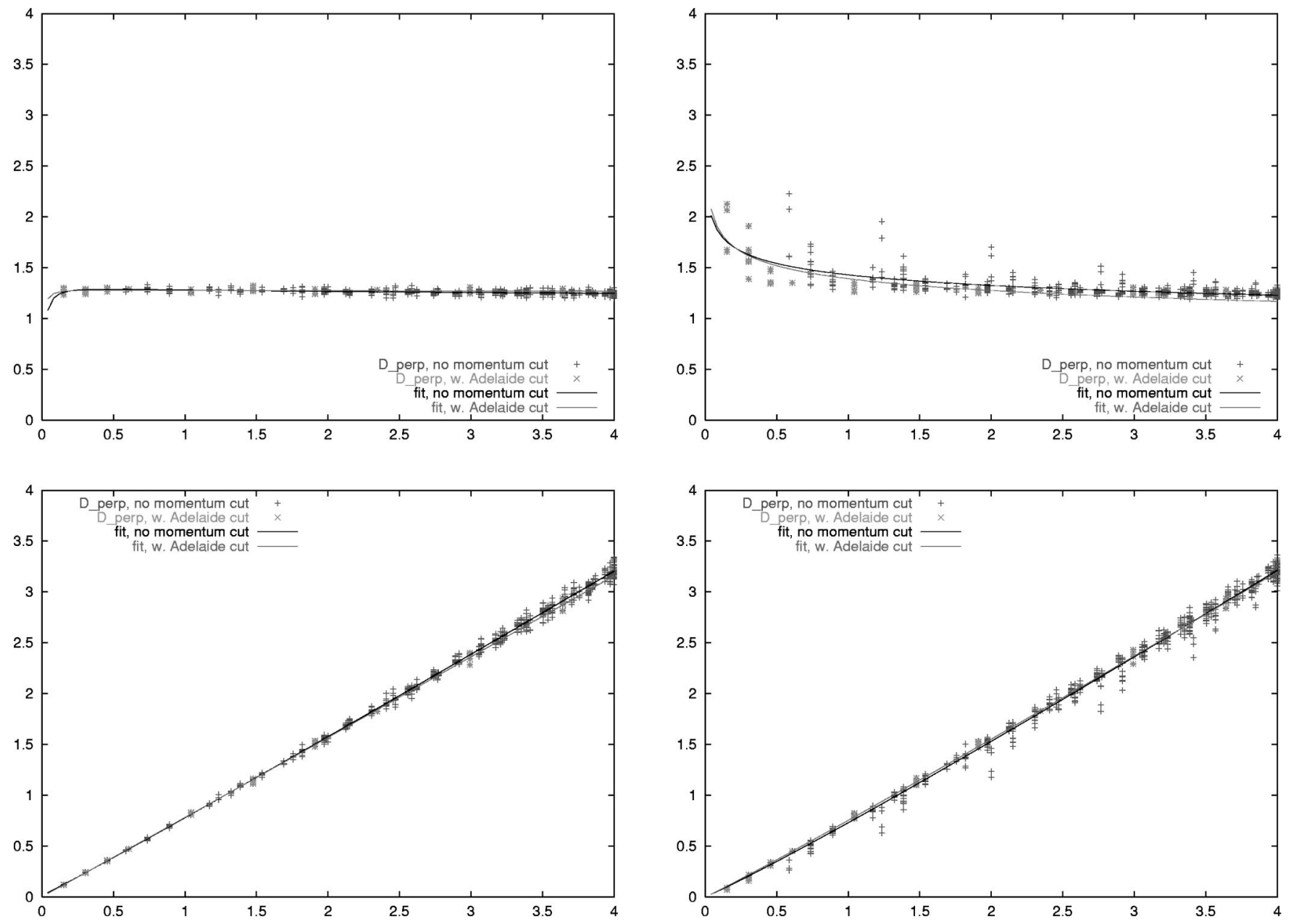

FIG. 2. Transverse component of the photon propagator at $\beta=1.05$ on a $16^{4}$ lattice, after modified iterative (left) and Laplacian (right) Landau gauge fixing. Since perturbatively $D_{\perp}\left(p^{2}\right) \simeq 1 / p^{2}$, the graphs $\hat{p}^{2} D_{\perp}\left(\hat{p}^{2}\right)$ vs $\hat{p}^{2}$ (top) and $1 / D_{\perp}\left(\hat{p}^{2}\right)$ vs $\hat{p}^{2}$ (bottom) are supposed to show an almost linear dependence. The modified ILG shows good rotational invariance, while the LLG shows a huge scatter of the data at a given $\hat{p}^{2}(+)$. After the cylindrical momentum cut $(\times)$ the scatter on the right hand side (LLG) is reduced (i.e., rotational invariance improves), but the IR enhancement in the Laplacian case persists.

If the propagator is transverse [i.e., $D_{\|}\left(\hat{p}^{2}\right)=0$ ], the transverse coefficient $D_{\perp}\left(\hat{p}^{2}\right)$ is simply obtained as $1 / 3$ of the trace of the propagator $C_{\mu \nu}(\hat{p})$.

In Fig. 2 we display our results for the Coulomb phase. Since in the weak coupling limit $D_{\perp}\left(p^{2}\right) \simeq 1 / p^{2}$, we plot $\hat{p}^{2} D_{\perp}\left(\hat{p}^{2}\right)$ vs $\hat{p}^{2}$ (top) and $1 / D_{\perp}\left(\hat{p}^{2}\right)$ vs $\hat{p}^{2}$ (bottom)—both of them after modified iterative (left) and Laplacian (right) gauge fixing. The striking fact is the huge amount of scatter in the data for the Laplacian case-data for a given $\hat{p}^{2}$ do not collapse on a universal line, i.e., rotational invariance is seriously broken after Laplacian fixing in the Coulomb phase. A simple way to elaborate on this point is to apply a cylindrical momentum cut [20], i.e., to decompose each momentum according to $\hat{p}=p_{\|} \hat{p}_{0}+\hat{p}_{\perp}$ where $\hat{p}_{0}=\frac{1}{2}(1,1,1,1)$, and to retain only those momenta with a component orthogonal to $(1,1,1,1)$ that is smaller than some constant, e.g., $\left|\hat{p}_{\perp}\right|^{2}$ $<0.2$. Those momenta out of the full set $(+)$ which survive this cut are plotted with crosses $(\times)$. For large momenta the scatter is considerably reduced, but for very low $\hat{p}^{2}$ the cut gets less effective and the IR enhancement by which the Laplacian correlator differs from its modified iterative counterpart is unchanged. Toward the end of this article evidence will be presented that the Laplacian gauge suffers from DDSs, and the present observation is consistent with that claim: If DDSs persist in the Laplacian ensemble, then one would expect rotational symmetry to be broken (remember that DDSs prefer to be planar) and low-lying momenta to be particularly affected, since DDSs represent large-scale objects.

In order to make contact with other studies, we fit the correlator to a functional form which allows for some IR enhancement; we use

$$
D_{\perp}\left(q^{2}\right)=Z \frac{m^{2 \alpha}}{q^{2(1+\alpha)}+m^{2(1+\alpha)}}
$$

with a "photon mass" $m$ and an "anomalous dimension" $\alpha$ (apart from the renormalization factor $Z$ ). Then our results may be stated as follows: In the Coulomb phase the modified 

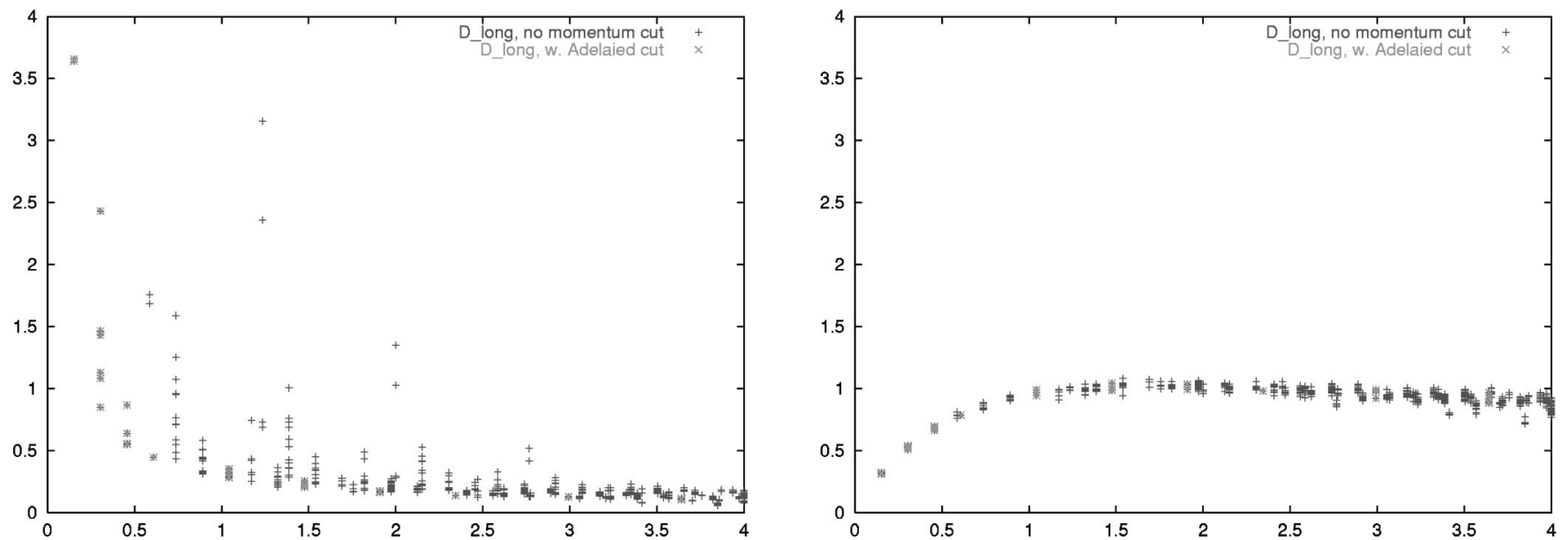

FIG. 3. The longitudinal part of the correlator (19), multiplied by $\hat{p}^{2}$, in the Laplacian gauge at $\beta=1.05$ (left) and at $\beta=0.95$ (right). This confirms earlier findings that the LLG does not yield a purely transverse propagator, but the longitudinal admixture seems to be much more severe (in particular for small momenta) in the Coulomb phase (left) than in the confined phase (right).

iterative prescription is consistent with a propagator (22) with zero mass and vanishing anomalous dimension, whereas the Laplacian gauge suggests a positive anomalous dimension.

An interesting point is to check the transversality of the propagator-we find it well obeyed in the modified ILG but seriously violated in the LLG. Figure 3 shows $\hat{p}^{2} D_{\|}$vs $\hat{p}^{2}$ for the Laplacian case both in the Coulomb phase (LHS) and in the confined phase discussed below.

To test whether the modified iterative and the Laplacian prescriptions differ on a deeper level (i.e., before the photon propagator is computed), we checked the distribution of link angles. They produce almost indistinguishible distributions (which are very close to a Gaussian centered about zero), i.e., they both comply with the perturbative preference for zero link angle.

\section{COMPARISON IN THE CONFINED PHASE}

We generate 1500 configurations on a $16^{4}$ lattice at $\beta$ $=0.95$, fix them with the Laplacian and the modified itera-

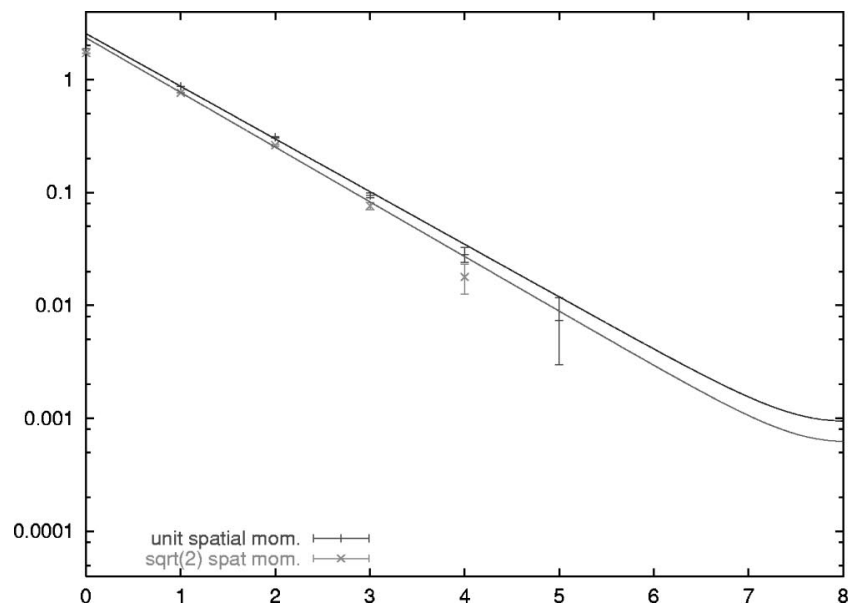

tive prescription, and compare the results for the correlator (19), (5).

Following the order of investigation in the Coulomb phase, we start with the correlator between gauge fields with 1 and $\sqrt{2}$ times the minimum spatial momentum. The result is presented in Fig. 4. With either procedure the two correlators seem to lie on top of each other, indicating that the effective mass (18) is dominated by the $m^{2}$ contribution. This confirms our expectation that there is no massless excitation in the disordered phase, but the two graphs show different slopes so that the resulting effective mass is a gaugedependent object. Moreover, it need not agree with what is usually called the photon mass, namely, the asymptotic mass in the $1^{+-}$plaquette-plaquette correlator [10], which is a gauge-invariant quantity. The gauge dependence of the effective mass of the correlator (19) for $\beta<\beta_{\mathrm{c}}$ is also evident from Fig. 5 and Table I below. Note finally that in the confined phase the Polyakov loop correlator is so noisy that in practice no signal can be extracted.

In order to investigate the difference between the two fix-

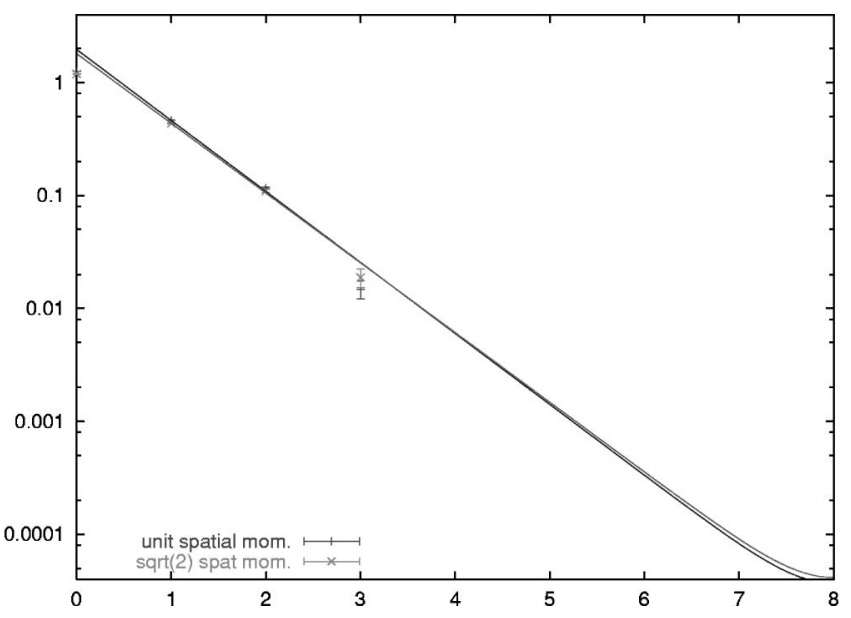

FIG. 4. Field (5) correlator with once $(+)$ or $\sqrt{2}$ times $(\times)$ the minimum spatial momentum at $\beta=0.95$. This yields information about two points in the dispersion relation (18). In the confined phase the Polyakov loop correlator is so noisy that no signal can be extracted. The effective mass in the modified iterative gauge (left) is smaller than that in the Laplacian gauge (right). 

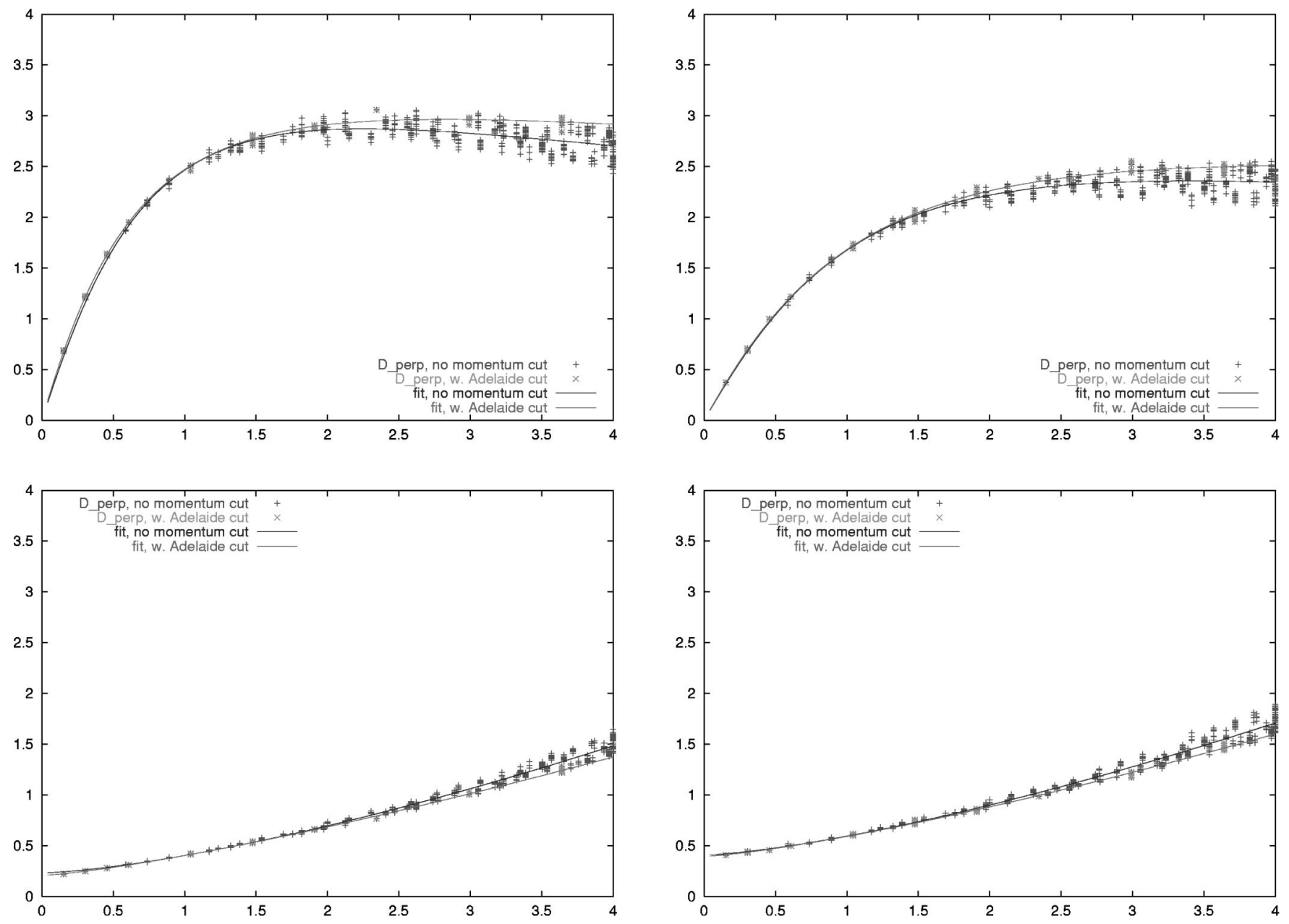

FIG. 5. Transverse component of the correlator (19) at $\beta=0.95$ on a $16^{4}$ lattice, after modified iterative (left) and Laplacian (right) Landau gauge fixing. We plot $\hat{p}^{2} D_{\perp}\left(\hat{p}^{2}\right)$ vs $\hat{p}^{2}$ (top) and $1 / D_{\perp}\left(\hat{p}^{2}\right)$ vs $\hat{p}^{2}$ (bottom). Both gauges yield a propagator that is not perfectly rotationally invariant, since there is a certain amount of scatter among the data for a given $\hat{p}^{2}(+)$. After the cylindrical momentum cut $(X)$ the scatter is much reduced. Note that the intercept, in the bottom line, of the correlator with the $y$ axis is larger in the Laplacian gauge than in the modified iterative case.

ing procedures, we go through the same steps as before: We consider the complete correlator (19) involving arbitrary four-momentum $\hat{p}_{\mu}$. The result for the confined phase is shown in Fig. 5. We plot $\hat{p}^{2} D_{\perp}\left(\hat{p}^{2}\right)$ vs $\hat{p}^{2}$ (top) and $1 / D_{\perp}\left(\hat{p}^{2}\right)$ vs $\hat{p}^{2}$ (bottom) — both after modified iterative (left) and Laplacian (right) fixing. Unlike in the Coulomb phase, rotational invariance is not a problem, i.e., the data for a given $\hat{p}^{2}$ collapse on a single line in either gauge. From a qualitative point of view it appears that in the confined phase
Eq. (19) is less sensitive to the details of the fixing procedure. On a quantitative level, however, the agreement is substantially less impressive. Again, we fit the correlators to the ansatz (22), which now seems much more adequate than in the ordered phase. The resulting parameters (see Table I) indicate that the confined phase is characterized by a distinctly nonzero mass parameter $m$ and a positive anomalous dimension $\alpha$, but the mass in the LLG is substantially larger than that in the modified ILG.

Again we check on the transversality. The modified iterative

TABLE I. Coefficients of the fits of $\hat{p}^{2} D_{\perp}\left(\hat{p}^{2}\right)$ vs $\hat{p}^{2}$ to Eq. (22) in the confined phase-see Fig. 5. The two lines represent the result without and with cylindrical momentum cut (see text). Note that the main uncertainty comes from the choice of the fitting method, not from statistics.

\begin{tabular}{lcccccc}
\hline \hline $\begin{array}{l}\beta=0.95 \\
\text { (confined) }\end{array}$ & $Z$ & $\begin{array}{c}\text { Modified ILG } \\
m\end{array}$ & $\alpha$ & $Z$ & LLG & $m$ \\
\hline All momenta & $5.25 \pm 0.04$ & $1.10 \pm 0.02$ & $0.41 \pm 0.01$ & $4.29 \pm 0.05$ & $1.33 \pm 0.02$ & $0.40 \pm 0.02$ \\
Cylindrical cut & $5.01 \pm 0.02$ & $1.02 \pm 0.01$ & $0.28 \pm 0.01$ & $4.29 \pm 0.02$ & $1.30 \pm 0.01$ & $0.29 \pm 0.01$ \\
\hline \hline
\end{tabular}



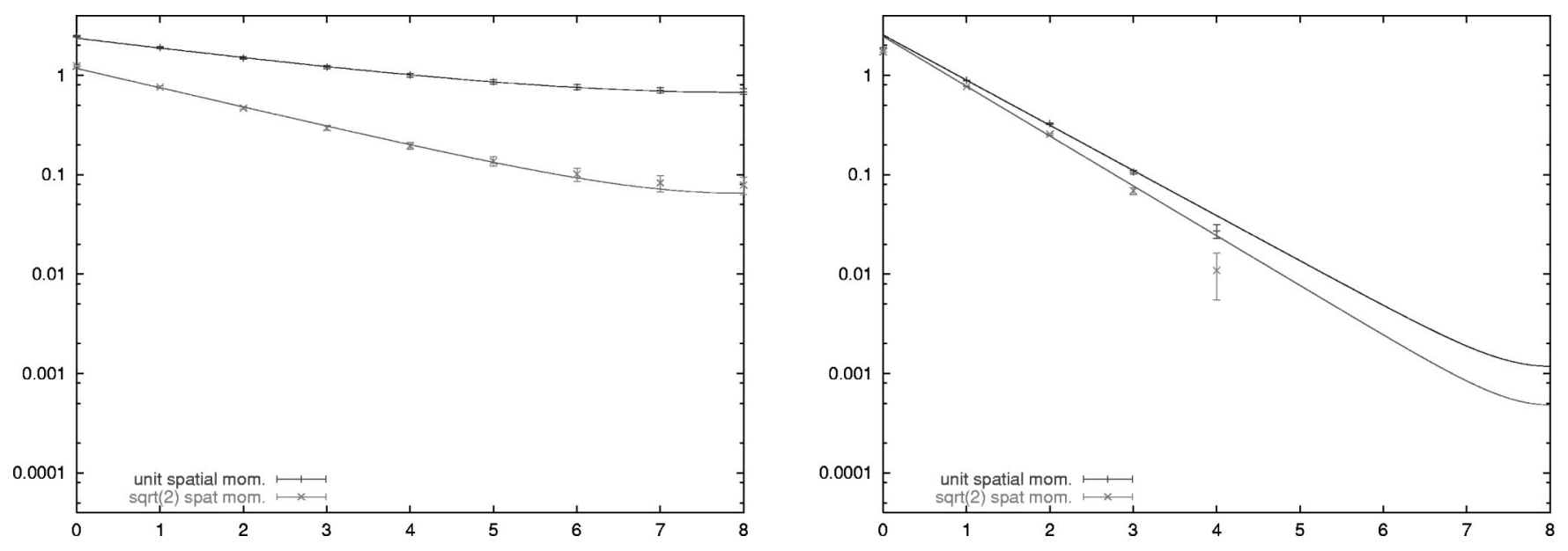

FIG. 6. Field (5) correlator with once $(+)$ or $\sqrt{2}$ times $(\times)$ the minimum spatial momentum in the "standard" iterative Landau gauge at $\beta=1.05$ (left) and at $\beta=0.95$ (right). The propagator in the Coulomb phase is flatter than its modified iterative or Laplacian counterparts at the same $\beta$ value (cf. Fig. 1), while in the confined phase it is in good agreement with its modified ILG counterpart but not with the Laplacian one (cf. Fig. 4).

correlator is (almost perfectly) transverse; the Laplacian one is not. However, the nontransversality in the LLG is seriously reduced compared to what we found in the Coulomb phase and rotational invariance is well respected in the transverse piece, too (see Fig. 3). In passing, we note that these findings are consistent with the hypothesis (which will be discussed below) that the Laplacian Landau gauge lacks the ability to fully remove the DDSs in the Coulomb phaserotational invariance is restored in the confined phase, since the DDSs percolate at the transition [17].

Finally, we check whether any difference between the two procedures may be seen in the distribution of the link angles, and here the answer is affirmative: In the confined phase, the distribution after Laplacian fixing deviates much more visibly from a Gaussian than that after iterative fixing.

\section{DISCUSSION AND CONCLUSION}

Having established that on either side of the phase transition in the compact U(1) theory the correlator (19) in the modified iterative Landau gauge differs from that in the Laplacian Landau gauge, it is natural to ask which are the specific properties of the gauge fixing procedures that cause these differences.

From the discussion of the gauge-fixing procedures in Sec. II, it is clear that double Dirac sheets and zeromomentum modes represent the primary candidates for gauge artifacts that might spoil the properties of a gauge (cf. [14]). Hence, a natural thing to try is to omit the DDS suppression in the iterative procedure while keeping the step which reduces the ZMM background; this is what we have introduced as our "standard" ILG. The result of such a gauge fixing is presented in Figs. 6 and 7. In the Coulomb phase, the correlators with minimum or next-to-minimum spatial momentum (LHS of Fig. 6) are flatter than those using modified ILG and LLG (cf. Fig. 1), indicating a negative $m^{2}$. This means that the DDSs tend to decrease the effective mass (18). In the confined phase the "standard" iterative correlators essentially agree with those in the modified iterative gauge. A more detailed picture is obtained by considering arbitrary four-momenta, as displayed in Fig. 7. The resulting longitudinal piece is essentially zero; hence only the transverse part is shown. The startling observation is that the "standard" ILG propagator shows the same amount of scatter and the same sort of IR enhancement in the Coulomb phase as we have seen in the case of the Laplacian correlator. On the other hand, in the confined phase the "standard" iterative propagator almost coincides with its modified counterpart. This is expected, since the DDSs percolate at the phase transition [17]; hence the efforts of the modified ILG to remove them are futile.

In an attempt to further substantiate the obvious suspicion that remnant DDSs are responsible for the abnormal IR behavior of the Laplacian propagator in the Coulomb phase and to shed light on the properties of the Laplacian gauge in the confined phase, we produce a scatter plot relating the value of the functional (7) to the remaining number of Dirac plaquettes in the gauge-fixed configuration. The result is shown in Fig. 8.

In the Coulomb phase the modified ILG always reaches the highest value of Eq. (7), among the three gauges considered. The "standard" ILG reaches (almost) the same extremum on the majority of configurations, but there is a considerable number of backgrounds on which it falls short quite drastically, and at the same time the total number of Dirac plaquettes is substantially increased. This correlation is paralleled in the LLG, but there the amount by which it falls short (in either respect) has a continuous spectrum. In other words, the "standard" ILG differs from the LLG by the gap in the functional value that separates "success" from "failure." Hence it appears that only a limited number of configurations causes (simultaneously for the Laplacian and the "standard" iterative procedure) a problem with respect to both the gauge functional and the number of Dirac plaquettes. We checked that these are, in most cases, the same configurations for both gauges. On our lattice the DDS 

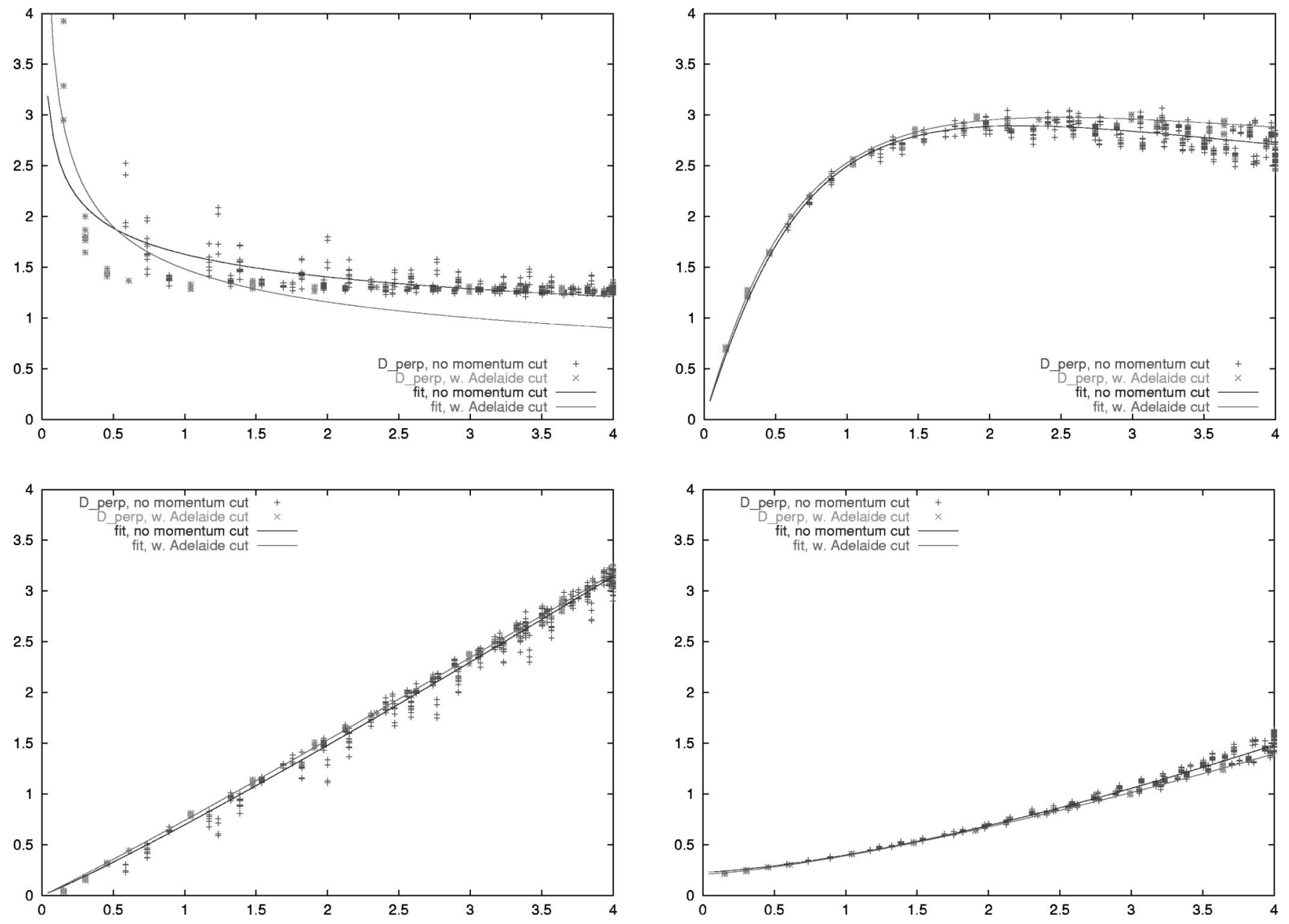

FIG. 7. Photon correlator in the "standard" iterative prescription at $\beta=1.05$ (left) and at $\beta=0.95$ (right). Each time the graphs for $\hat{p}^{2} D_{\perp}\left(\hat{p}^{2}\right)$ vs $\hat{p}^{2}$ (top) and $1 / D_{\perp}\left(\hat{p}^{2}\right)$ vs $\hat{p}^{2}$ (bottom) are shown. In the Coulomb phase the remaining DDSs cause a massive IR enhancement which is absent in the modified ILG counterpart but equally pronounced in the LLG (cf. Fig. 2). In the confined phase the result agrees with the modified ILG correlator (cf. Fig. 5).

condition (9) reads $2 \times 16^{2}=512$ per plane. With a typical number of $O(2000)$ Dirac plaquettes at $\beta=1.05$ in a modified ILG configuration, one would expect a configuration with a DDS sheet to have more than $O$ (2500) Dirac plaquettes. This is precisely where the gap in the number of Dirac plaquettes for "standard" iterative fixing ends. Since the Laplacian fixing shows a continuous excess in the number of Dirac plaquettes, this correlation analysis cannot prove that DDSs present a problem to the LLG procedure. However, the condition (9) being met, together with our previous observation that rotational invariance is seriously broken (in particular for small momenta; see Fig. 3) is strong circumstantial evidence.

In the confined phase things look much simpler: Again, the modified ILG wins the competition for the extremization of the gauge functional (7). The "standard" ILG is in second rank, but it is very close-with respect to both the functional value and the number of remnant Dirac plaquettes. The LLG is far off-again with respect to both criteria. The only surprise is by how much it misses the extremum and by how many Dirac plaquettes it deviates from the modified ILG. Again this establishes a link to our previous finding that the
LLG respects rotational invariance in the confined phase: If unresolved DDSs in the Coulomb phase are responsible for the shortcomings of the Laplacian gauge, then one would expect, since the DDSs percolate at the transition [17], that rotational invariance gets restored in the confined phase while the excess of Dirac plaquettes remains-and this is precisely what we see.

While these preliminary observations clearly show a demand for future research, we would like to close with a few general remarks.

At first sight, it looks as if some of our correlators are quite "fuzzy." Unlike many other groups we have refrained from averaging over momenta with the same $\hat{p}^{2}$; if one took the average $D_{\perp}\left(\hat{p}^{2}\right)$, then all propagators would become thin lines. However, as is obvious from the upper right plot in Fig. 2, such a procedure would only reveal a slight IR enhancement, and the information about the lack of rotational invariance would be lost. Hence we believe it is important to retain the full information about the spread of correlator points associated with a given $\hat{p}^{2}$.

It is not clear whether the qualitative aspects of the propa- 

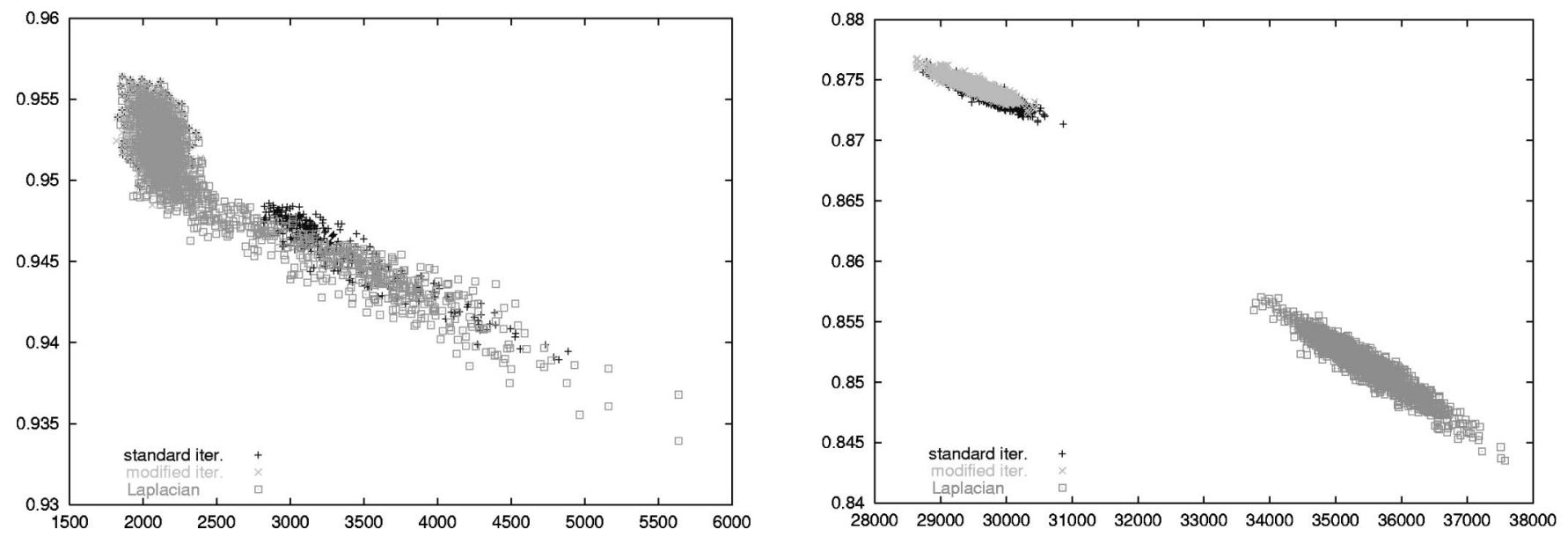

FIG. 8. Correlation between the final value of the functional (7) and the number of Dirac plaquettes in the fixed configuration in the Coulomb phase (left) and in the confined phase (right).

gators we have studied on a $16^{4}$ grid stay invariant under a change of the lattice size. On one hand the ability of the ILG to remove DDSs through repeated attempts will degrade drastically on larger lattices; presumably the cutoff on the outer loop (as discussed in Sec. II) should be increased exponentially with the volume. On the other hand, DDSs on a larger lattice involve more Dirac plaquettes and become less frequent by purely entropic suppression. Which effect wins is unclear. Preliminary studies on a $32^{4}$ grid indicate that the Laplacian correlator might be much better behaved on that lattice size-unfortunately the available statistics has prevented us from reaching any conclusion. Still, our $16^{4}$ lattice is much larger than those used in previous investigations of the modified ILG [14].

After dealing with so many details of which quantity correlates with which other one, we wish to emphasize a simple point: We have presented evidence that all three gauges considered yield smooth configurations in either phase. At $\beta$ $=1.05$ we see no differences in the distribution of link angles at all, whereas at $\beta=0.95$ the configurations tend to be slightly smoother with both types of iterative fixing than in the Laplacian gauge. Notably, in spite of the distribution of link angles being almost indistinguishable, the propagators (19) on these ensembles may be strikingly different.
The inability of several gauge-fixing procedures to reproduce the known perturbative behavior in the Coulomb phase of the compact $\mathrm{U}(1)$ theory raises concerns about the validity of results for $\mathrm{SU}(2)$ or $\mathrm{SU}(3)$ in either the standard iterative or the Laplacian gauge. If a U(1) subgroup may already cause a problem, one does not feel inclined to blindly trust the result of any fixing procedure in the non-Abelian case. The mass that we have extracted from the gauge-fixed field correlator is not gauge invariant (see Table I). This feature is likely to persist in the non-Abelian case below $T_{c}$. Therefore, the quantitative meaning of gauge-fixed gluon mass measurements should be considered with caution. It will be interesting to see whether our observation that the deconfined phase is more sensitive to the details of the fixing procedure carries over to the non-Abelian case, too.

\section{ACKNOWLEDGMENTS}

Out of the vast body of institutional addresses, one deserves to be highlighted: The Institute for Nuclear Theory is where the two of us met during the program "Lattice QCD and Hadron Phenomenology" (INT-01-3) and the stimulating atmosphere genuine to this place spurred us on to complete the current project. One of us (S.D.) acknowledges useful discussions with Urs Heller and Karl Jansen.
[1] F.D. Bonnet et al., Nucl. Phys. B (Proc. Suppl.) 83, 905 (2000).

[2] V.N. Gribov, Nucl. Phys. B139, 1 (1978).

[3] J.C. Vink and U.J. Wiese, Phys. Lett. B 289, 122 (1992); J.C. Vink, Phys. Rev. D 51, 1292 (1995); A.J. van der Sijs, Nucl. Phys. B (Proc. Suppl.) 53, 535 (1997); Prog. Theor. Phys. Suppl. 131, 149 (1998).

[4] A.J. van der Sijs, Nucl. Phys. B (Proc. Suppl.) 73, 548 (1999); C. Alexandrou, P. de Forcrand, and E. Follana, Phys. Rev. D 63, 094504 (2001); 65, 114508 (2002); 65, 117502 (2002); P. de Forcrand and M. Pepe, Nucl. Phys. B598, 557 (2001); A. Montero, Phys. Lett. B 517, 142 (2001).

[5] A.H. Guth, Phys. Rev. D 21, 2291 (1980); J. Fröhlich and T.
Spencer, Commun. Math. Phys. 83, 411 (1982).

[6] M. Creutz, L. Jacobs, and C. Rebbi, Phys. Rev. D 20, 1915 (1979); J. Jersak, C.B. Lang, and T. Neuhaus, Phys. Rev. Lett. 77, 1933 (1996).

[7] L.A. Fernandez, A. Munoz Sudupe, R. Petronzio, and A. Tarancon, Phys. Lett. B 267, 100 (1991); G. Bhanot, T. Lippert, K. Schilling, and P. Überholz, Nucl. Phys. B378, 633 (1992); I. Campos, A. Cruz, and A. Tarancon, Phys. Lett. B 424, 328 (1998); Nucl. Phys. B528, 325 (1998); G. Arnold, T. Lippert, K. Schilling, and T. Neuhaus, Nucl. Phys. B (Proc. Suppl.) 94, 651 (2001).

[8] T.P. Killingback, Phys. Lett. 138B, 87 (1984). 
[9] P. Coddington, A. Hey, J. Mandula, and M. Ogilvie, Phys. Lett. B 197, 191 (1987).

[10] B. Berg and C. Panagiotakopoulos, Phys. Rev. Lett. 52, 94 (1984); J. Cox, W. Franzki, J. Jersak, C.B. Lang, T. Neuhaus, and P.W. Stephenson, Nucl. Phys. B499, 371 (1997).

[11] A. Nakamura and M. Plewnia, Phys. Lett. B 255, 274 (1991).

[12] P. de Forcrand and R. Gupta, Nucl. Phys. B (Proc. Suppl.) 9, 516 (1989).

[13] D. Zwanziger, Phys. Lett. B 257, 168 (1991); Nucl. Phys. B364, 127 (1991).

[14] I.L. Bogolubsky, V.K. Mitrjushkin, M. Müller-Preussker, and
P. Peter, Phys. Lett. B 458, 102 (1999).

[15] T.A. DeGrand and D. Toussaint, Phys. Rev. D 22, 2478 (1980).

[16] V. Grosch et al., Phys. Lett. 162B, 171 (1985).

[17] M. Baig, H. Fort, and J.B. Kogut, Phys. Rev. D 50, 5920 (1994); M. Baig and J. Clua, ibid. 57, 3902 (1998).

[18] P. van Baal, Nucl. Phys. B (Proc. Suppl.) 42, 843 (1995); J.E. Mandula, ibid. 106, 998 (2002).

[19] See http://www.caam.rice.edu/software/ARPACK

[20] D.B. Leinweber, J.I. Skullerud, A.G. Williams, and C. Parrinello, Phys. Rev. D 60, 094507 (1999); 61, 079901(E) (1999). 